\title{
Long non-coding RNA PVT1 promotes proliferation and migration of pulmonary smooth muscle cells in pulmonary arterial hypertension through miR-140- 5p/Fxr1 axis
}

Botao Zhao

Cangzhou Cenrtal Hosptial

Yuchao Shen

Cangzhou Central Hospital

Qinghua Wu

Cangzhou Central Hospital

Li Yao

Cangzhou Central Hospital

Yamin Hu ( $\sim$ huyamin091@163.com)

Cangzhou Central Hospital https://orcid.org/0000-0003-4299-7745

\section{Research}

Keywords: pulmonary arterial hypertension, pulmonary smooth muscle cells, proliferation, migration, PVT1, miR-140-5p, Fxr1

Posted Date: July 17 th, 2020

DOI: https://doi.org/10.21203/rs.3.rs-43614/v1

License: (c) (i) This work is licensed under a Creative Commons Attribution 4.0 International License.

Read Full License 


\section{Abstract}

Background: Pulmonary arterial hypertension (PAH) is a devastating disease and long non-coding RNAs (IncRNAs) are essential for PAH progression. Also, IncRNA plasmacytoma variant translocation 1 (PVT1) plays important roles in cell proliferation and migration, however, the effect of PVT1 on pulmonary artery smooth muscle cells (HPASMCs) in PAH remains unknown. This study aimed to determine the role and underlying mechanisms of PVT1 in HPASMCs.

Methods: The results demonstrated that the expression of PVT1 was upregulated in pulmonary arterial tissues and HPASMCs and was positively correlated with pulmonary arterial pressure.

Results: Knockdown and overexpression of PVT1 suppressed and promoted HPASMCs proliferation and migration, respectively. Mechanical and functional studies revealed that PVT1 functioned as a competing endogenous RNA of miR-140-5p and their expressions were negatively correlated.

Conclusions: Knockdown of PVT1 did not suppress proliferation and migration in HPASMCs transfected with the miR-140-5p inhibitor. Moreover, fragile X-related proteins 1 (Fxr1) was identified as the target gene of miR-140-5p. Downregulation of PVT1 decreased the level of Fxr1 while this inhibitory effect was reversed by the miR-140-5p inhibitor. In conclusion, the upregulation of PVT1 promoted proliferation and migration in HPASMCs though the miR-140-5p/Fxr1 signaling pathway, indicating PVT1 may serve as a promising diagnostic biomarker for $\mathrm{PAH}$.

\section{Introduction}

Pulmonary arterial hypertension $(\mathrm{PAH})$ is a fatal disease lacking effective early diagnosis and treatments [1]. Also, PAH is characterized by aberrant functions and/or structures of the pulmonary artery smooth muscle cells (PASMCs), endothelial cells, vascular fibroblasts, and inflammatory cells, leading to vascular stiffening, vasoconstriction, and vascular lumen loss [2]. This devastating vascular remodeling results in pulmonary vascular resistance and promotes right ventricular failure and death [3]. Although PAH is a rare pulmonary disorder, $\mathrm{PAH}$ has been widely regarded as a severe and poor prognosis disease.

Therefore, it is worthwhile to explore the mechanisms underlying the pathological progression of PAH, particularly in vascular remodeling, which would facilitate the development of novel diagnostic and treatment strategies for $\mathrm{PAH}$.

In PAH, pulmonary vessel wall remodeling, vasoconstriction, and thrombosis causes elevated pulmonary vascular resistance and narrowing [4]. Pulmonary vascular remodeling is a complicated process associated with multiple layers of the vessel wall and cellular heterogeneity in the pulmonary arterial wall [5]. In general, all types of PAH remodeling are characterized by the presence of a layer of smooth muscle cells in small peripheral, nonmuscular pulmonary arteries within the respiratory acinus [6]. Accordingly, such aberrant smooth muscle cell proliferation ultimately results in chronic obstruction of the small pulmonary arteries [7]. In addition, it has been demonstrated that vascular remodeling is the consequence of an imbalance between apoptosis and proliferation in smooth muscle cells, manifesting upregulated 
proliferation [8]. Collectively, the cellular activity of smooth muscles is tightly associated with pulmonary vascular remodeling and the progression of PAH.

Long non-coding RNAs (IncRNAs) is defined as a group of non-protein-coding RNAs over 200 nucleotides in length [9]. It has been well-documented that IncRNAs play essential roles in gene expression through multiple mechanisms, such as transcription modulation, post-transcriptional processing, and chromosome remodeling [10]; thus, IncRNAs are crucial regulators for diverse molecular and cellular activities, impacting many physiological and pathological processes [11]. The IncRNA plasmacytoma variant translocation 1 (PVT1) is encoded by the human PVT1 gene and is involved in several important molecular mechanisms, including encoding microRNAs, regulating transcription factor myelocytomatosis (MYC), and DNA rearrangement [12]. In addition, the aberrant expression of PVT1 is a promising marker for predicting progression and prognosis of several tumor types [13], such as, gallbladder cancer [14], colorectal cancer [15], and lung cancer [16, 17]. Furthermore, PVT1 promotes angiogenesis of vascular endothelial cells by influencing cell proliferation and migration [18]. Together, PVT1 is an important regulator contributing to various cellular activities, however, its role in pulmonary vascular remodeling, to date, has not been reported. Therefore, the objective of this study was to determine the role of PVT1 in proliferation and migration of human PASMCs (HPASMCs) in PAH and its related mechanisms. The observations of this study may provide novel therapeutic biomarkers for predicting the progression and prognosis of PAH.

\section{Materials And Methods}

\section{Patient and sample information}

In the study, all patients were informed before their inclusion and written consents of patients were obtained. All experiment protocols were approved by the Ethics Committee of Cangzhou Central Hospital (Cangzhou, China) and experimental procedures were conducted according to the Declaration of Helsinki Principles [19]. Fourteen patients (22-59 years old; 6 males and 8 females) diagnosed with PAH had received lung transplantation surgery at Cangzhou Central Hospital between May 2016 and May 2018. The information about patients with PAH and healthy control donors was summarized in Table 1. Before the surgical procedure, mean pulmonary artery pressure (mPAP) was measured through echocardiographic examinations (PHILIPS iE Elite; PHLIPS, Amsterdam, Netherlands). The 6-min walk test (6 MWT) of patients were between 100 and $530 \mathrm{~m}$. PAH diagnosis were verified by echocardiography and the diagnostic procedures were performed accoding to European Society of Cardiology and European Respiratory Society guidelines for the diagnosis of pulmonary arterial hypertension (2015) [20]. Patients with the following conditions were excluded from this study, including psychosis, severe obstructive pulmonary disease, chronic liver disease, chronic kidney disease, amyloidosis, portal hypertension, drug addiction history and so on. Meanwhile, patients who had taken prostacyclin, L-arginine, sildenafil, and endothelin receptor antagonist were also excluded. In this study, healthy lung tissue samples were donored by patients who were not suitable for transplantation. The inclusion criteria for the donors of healthy lung tissues were as follows: $<55$ years old; $<20$ packs of cigarattes consumption per year; no 
chest trauma; no sustained mechanical ventilation; positive end-expiratory pressure (PEEP), $5 \mathrm{~cm}$; the fraction of inspired oxygen (FiO2), 1.0; partial pressure of oxygen (PaO2), > $300 \mathrm{mmHg}$; clear lung fields shown in the chest films; and a clear trachea conformed by bronchoscopy.

Table 1

Characteristics of healthy donor and patients with PAH

\begin{tabular}{|llllll|}
\hline Patient ID & Sex & Age & Ethnicity & Diagnosis & mPAP $(\mathrm{mm} / \mathrm{Hg})$ \\
\hline PAH-1 & M & 41 & Asian & HPAH & 89 \\
\hline PAH-2 & M & 36 & Asian & HPAH & 93 \\
\hline PAH-3 & M & 53 & Asian & HPAH & 90 \\
\hline PAH-4 & M & 36 & Asian & HPAH & 92 \\
\hline PAH-5 & M & 51 & Asian & HPAH & 94 \\
\hline PAH-6 & M & 56 & Asian & HPAH & 97 \\
\hline PAH-7 & F & 22 & Asian & HPAH & 95 \\
\hline PAH-8 & F & 39 & Asian & HPAH & 98 \\
\hline PAH-9 & F & 54 & Asian & HPAH & 95 \\
\hline PAH-10 & F & 53 & Asian & HPAH & 100 \\
\hline PAH-11 & F & 55 & Asian & HPAH & 115 \\
\hline PAH-12 & F & 51 & Asian & HPAH & 111 \\
\hline PAH-13 & F & 56 & Asian & HPAH & 108 \\
\hline PAH-14 & F & 29 & Asian & HPAH & 105 \\
\hline PAH-15 & F & 47 & Asian & HPAH & 89 \\
\hline PAH-16 & F & 59 & Asian & HPAH & 93 \\
\hline Healthy Control & M & 44 & Asian & Donor lung tissue & 20 \\
\hline Healthy Control & M & 47 & Asian & Donor lung tissue & 22 \\
\hline Healthy Control & M & 58 & Asian & Donor lung tissue & 23 \\
\hline Healthy Control & F & 39 & Asian & Donor lung tissue & 24 \\
\hline Healthy Control & F & 53 & Asian & Donor lung tissue & 24 \\
\hline Healthy Control & F & 41 & Asian & Donor lung tissue & 20 \\
\hline HPAH: hereditary pulmonary artery hypertension & \\
\hline
\end{tabular}




\section{Preparation of lung tissues and HPASMCs}

The PAH lung tissues were collected from patients who had received lung transplantations at Cangzhou Central Hospital (Cangzhou, China). Healthy lung tissues were collected from participants who were not suitable for lung transplantation and were used as the control in this study. The pulmonary artery (PA) tissues preparation procedure was conducted as previously described [21,22]. Briefly, a small piece of distal PA $(<1,000 \mu \mathrm{m}$ diameter) were microdissected from explanted PAH from 4 patients and healthy lung tissues were collected from 3 health donors. PAs were first digested in Hank's Balanced Salt Solution (HBSS) (Thermo Fisher Scientific, Shanghai, China) supplemeted with collagenase (type II) and deoxyribonuclease (DNase). After isolating epithelial layer, the smooth muscles were further diested in HBSS supplemented with $1.5 \mathrm{mg} / \mathrm{ml}$ bovine Serum Albumin (BSA), $0.5 \mathrm{mg} / \mathrm{ml}$ elastase, and $2 \mathrm{mg} / \mathrm{ml}$ collagenase. Then, HPASMCs were cultured in SmGM-2 Smooth Muscle Growth medium-2 BulletKit media (Lonza Group, Ltd., Houston, USA) supplemented with 10\% (v/v) heat-inactivated fetal bovine serum (FBS; Gibco, Grand Island, USA), human recombinant fibroblast growth factor ( $3 \mathrm{ng} / \mathrm{ml})$, gentamicin $(40 \mu \mathrm{g} / \mathrm{ml})$, insulin $(4 \mu \mathrm{g} / \mathrm{ml})$, and human recombinant epidermal growth factor $(0.5 \mathrm{ng} / \mathrm{ml})$ at $37^{\circ} \mathrm{C}$ in a humidified atmosphere with $5 \% \mathrm{CO}_{2}$. The HPASMCs (4-6 passages) were used for subsequent experiments. Each treatment group consisted of 3-5 replicates.

\section{Hematoxylin and eosin (H\&E) staining}

The histopathological features of lung tissue samples were assessed by H\&E staining. Briefly, lung tissues were sectioned into blocks ( $5 \mu \mathrm{m}$ thickness) and fixed in $10 \%(\mathrm{w} / \mathrm{v})$ neutral-buffered formalin at $4{ }^{\circ} \mathrm{C}$ overnight. Next, tissue sections were dehydrated with a graded ethanol series, cleaned, and embedded in paraffin. Sections were stained with hematoxylin for 10 minutes and then eosin for 5 minutes. Lung tissue slices were imaged by light microscopy.

\section{Immunofluorescence assay}

The purity of HPASMCs was assessed by immunofluorescence assays which detect the expression of the smooth muscle myosin heavy chain. The HPASMCs were placed on glass cover slips and fixed with $4 \%$ polyformaldehyde for 15 minutes. Slices were then incubated with smooth muscle myosin heavy chain (SM-MHC) antibody (1:400; Abcam, Cambridge, USA). Slices were imaged by confocal laser scanning microscopy (Leica, Solms, Germany).

\section{Cell transfection}

The PVT1 shRNA sequence and the miR-140-5p mimic and inhibitor were obtained from Shanghai GenePharma Co., Ltd (Shanghai, China). The plasmid pcDNA-PVT1 were synthesized by Shanghai GenePharma Co., Ltd (Shanghai, China). The sh-PVT1 (30 nmol/l), miR-140-5p mimic (50 $\mu \mathrm{M})$, and miR140-5p inhibitor $(50 \mu \mathrm{M})$ were transfected into HPASMCs based on the manufacturer's instructions using the Lipofectamine ${ }^{\mathrm{TM}} 3000$ Transfection Reagent (Invitrogen, Waltham, USA). After 24 hours of transfection, the HPASMCs were used for subsequent experiments.

\section{Real-time PCR}


Total RNA was extracted from lung tissues and HPASMCs using the TRIzol reagent (Invitrogen, CA, USA). First strand cDNAs were synthesized using the M-MLV Reverse Transcriptase (RNase H) kit (GeneCopoeia, Rockville, USA). Real-time PCR was performed using the ABI StepOne Real-Time PCR System (Applied Biosystems, Foster City, USA) with recommended reaction conditions in the manufacturer's instructions. The primers were synthesized by Shanghai GenePharma Co., Ltd (Shanghai, China). The amplification efficiency of primers was validated before use and the efficiency were 95-105\%. Data were analyzed using the $2^{-\triangle \triangle C t}$ method [23]. GAPDH and U6 were used as reference genes.

\section{Western blots}

Total protein was isolated from lung tissues and HPASMCs using cell lysis buffer (Thermo Fisher Scientific, Shanghai, China). Western blots were performed as previously described [24]. The primary antibodies against proliferating cell nuclear antigen (PCNA, 1:1000), fragile X-related proteins 1 (Fxr1, 1:1000), cyclin A1 (1:1000), cyclin D1(1:1000), cyclin E1 (1:1000), and $\beta$-actin (1:5000) were obtained from Santa Cruz (Santa Cruz, Shanghai, China). Goat-anti-rabbit IgG secondary antibody (1:10000) was used as the secondary antibody (Santa Cruz, Shanghai, China). The quantification of optical density for protein bands was evaluated using the Imagej software [25].

\section{CCK-8 assay}

The HPASMCs $\left(1 \times 10^{5}\right.$ cells/well) were incubated in a 96-well cell culture dish without serum for 24 hours. Cell proliferation was then assessed at 0, 24, 48, 72, and 96 hours using the Cell Counting Kit 8 (Abcam, Cambridge, USA) according to the manufacturer's instructions. The absorbance OD values were obtained at $450 \mathrm{~nm}$.

\section{Flow cytometer assay}

The cell cycle was assessed using flow cytometer assays (FACSort; Becton Dickinson) according to the manufacturer's instructions. Data were analyzed using the ModFit software (Verity Software House,Topsham, USA).

\section{Bioinformatics and luciferase reporter assay}

TargetScan (www.targetscan.org) and RNAhybrid (http://bibiserv.techfak.uni-bielefeld.de/) were used to predict putative target genes. The luciferase vectors including wild-type or mutant 3'UTR of PVT1 containing the miR-140-5p binding site were purchased from Shanghai GenePharma Co., Ltd (Shanghai, China). Also, the 3'UTR of Fxr1 containing the miR-140-5p binding site was synthesized and inserted into pmirGLO Dual-Luciferase miRNA Target Expression Vector (Promega, Madison, WI, USA). Each engineered luciferase reporter vetors mentioned above and miR-140-5p mimic/ miR-140-5p mimic negative control (NC) were co-transfected to HPASMCs using the Lipofectamine ${ }^{\text {TM }} 3000$ Transfection Reagent (Invitrogen, Waltham, USA) according to the manufacturer's instructions. After 8 hours, the transfection media were removed and HPASMCs were cultured in SmGM-2 Smooth Muscle Growth medium-2 BulletKit media (Lonza Group, Ltd., Houston, USA) for 24 hours. Luciferase activity was 
assessed using the Dual-Light Chemiluminescent Reporter Gene Assay System (Applied Biosystems, Foster City, USA) and normalized by Renilla luciferase activity

\section{RNA Binding Protein Immunoprecipitation (RIP) assay}

The physical interaction between PVT1 and miR-140-5p was determined using RIP assay kits (SigmaAldrich Corporation, St. Louis, USA) according to the manufacturer's instructions. The detailed procedure was previously described [26].

\section{Wound-healing migration assay}

After transfection, HPASMCs were subjected to the wound-healing assay and the detailed procedure was previously described [24].

\section{Statistical analysis}

Data were presented as mean \pm standard error of mean. Statistical analysis was performed using SPSS v.19.0 software (SPSS, IL, USA). The correlations between PVT1 expression and clinicopathological features, PVT1 expression, and miR-140-5p level were analyzed with the Pearson's correlation. Differences between groups were analyzed with Student's t-test or one-way ANOVA. Differences were regarded to be statistically significant at $P<0.05$. In this study, each treatment group consisted of 3-5 replicates.

\section{Results}

\section{Upregulation of PVT1 in PA tissues and HPASMCs}

In this study, the vascular wall morphology of healthy and PAH lung tissues was assessed. The H\&E staining assay revealed that the vascular wall thickness of PAH patients was larger than those of healthy individuals (Fig. 1.A). The immunofluorescence assay was used to verify the purity of HPASMCs by detecting the smooth muscle myosin heavy chain (Fig. 1.B). Moreover, PVT1 exerts essential roles in various tumor types, promoting proliferation and inhibiting apoptosis [12]. Some common cellular properties, such as high proliferative levels, are observed in both HPASMCs and tumor cells [22, 23]. Therefore, PVT1 may also participate in the progression of PAH, particularly vascular remodeling. To verify this hypothesis, the expression of PVT1 in both PA tissue samples and HPASMCs were detected. The results showed that the level of PVT1 was higher in PA tissue samples (Fig. 1.C) and HPASMCs (Fig. 1.D) compared with those in the control group. As one of primary pathological features of PAH, the pulmonary artery pressure of PAH patients was significantly higher than those of healthy participants (Fig. 1.E). Furthermore, the expression of PVT1 was positively correlated with pulmonary artery pressure (Fig. 1.F), suggesting that PVT1 may serve as a diagnostic marker for PAH. Taken together, upregulated expression of PVT1 may be involved in the development and progression of PAH. 


\section{PVT1 modulated the viability, proliferation, and migration of HPASMCs}

To further determine the roles of PVT1 in HPASMCs, sh-PVT1 and pcDNA-PVT1 were applied to knockdown and overexpress PVT1 in HPASMCs, respectively. The transfection efficiencies were assessed using real-time PCR (Fig. 2.A and Fig. 3.A). In CCK-8 assays, HPASMCs transfected sh-PVT1 displayed lower cell viability whereas the higher cell viability was found in HPASMCs transfected with pcDNA-PVT1 compared to the control group (Fig. 2.B and Fig. 3.B). In addition, the flow cytometry assay revealed that downregulation and upregulation of PVT1 decreased and increased the proportion of cells in the $S$ and $\mathrm{G}_{2}$ phases, respectively (Fig. 2.C and Fig. 3.C). To confirm the role of PVT1 in cell proliferation, protein expressions of several cell proliferation protein markers were detected, including PCNA, Cyclin A1, Cyclin D1, and Cyclin E1 $[27,28]$. The results showed that reduced PVT1 level was associated with decreased expressions of PCNA, Cyclin A1, Cyclin D1, and Cyclin E1 while elevated PVT1 exerted opposite roles to these cell proliferation markers compared to the control group (Fig. 2.D and Fig. 3.D). Furthermore, using the wound-healing migration assay, the knockdown and overexpression of PVT1 inhibited and promoted HPASMCs migration ability, respectively (Fig. 2.E and Fig. 3.E). Collectively, PVT1 plays important regulator roles in HPASMCs viability, proliferation, and migration, which suggests that there is an essential involvement of PVT1 in PAH progression.

\section{PVT1 regulated HPASMCs activities through sponging miR- 140-5p}

To further determine the mechanisms underlying the effect of PVT1 in PAH, the miRNA associated with the function of PVT1 in the progression of PAH was identified. Bioinformatics analysis revealed that a putative binding site of miR-140-5p was found in the 3'UTR of PVT1 (Fig. 4. H). Thus, the expression of miR-140-5p by real-time PCR was detected and the results showed that miR-140-5 $p$ was downregulated in PA tissues and HPASMCs (Fig. 4.A and B). Also, the downregulation and upregulation of PVT1 increased and decreased the expression of miR-140-5p in HPASMCs, respectively (Fig. 4.C and D). The miR-140-5p mimic and inhibitor inhibited and elevated the level of PVT1, respectively (Fig. 4. E). The expression of PVT1 was negatively correlated with miR-140-5p expression (Fig. 4. F). These results together revealed that PVT1 may act as a competing endogenous RNA (ceRNA) to miR-140-5p. To further verify the interaction between PVT1 and miR-140-5p, luciferase reporter assays showed that HPASMCs cotransfected with the wide-type PVT1 plasmid and miR-140-5p mimic reduced the luciferase activity while the luciferase activity was not affected by co-transfection of the mutant PVT1 plasmid (Fig. 4.I). Moreover, the physical interaction between PVT1 and miR-140-5p was also found in the RIP assay where both PVT1 and miR-140-5p were enriched in AGO2 immunoprecipitates compared to the control (Fig. 4.J). Thus, these results suggest that PVT1 functions as a ceRNA for miR-140-5p in HPASMCs. 


\section{MiR-140-5p directly targeted Fxr1 in PA tissues and HPASMCs}

To determine the downstream regulator of miR-140-5p in PAH, bioinformatics analysis was applied to predict the putative target gene of miR-140-5p. The results showed that a binding site of miR-140-5p was observed in 3'UTR of Fxr1 (Fig. 5.G). Subsequently, the luciferase reporter assay confirmed this prediction, where HPASMCs co-transfected with wild-type plasmid of Fxr1 and miR-140-5p mimic had reduced luciferase activity while the luciferase activity did not alter in HPASMCs transfected with the mutant Fxr1containing plasmid (Fig. 5.H). Also, functional studies demonstrated that the miR-140-5p mimic and inhibitor inhibited and enhanced the protein expression of Fxr1 in HPASMCs, respectively (Fig. 5. A and B). The transfection efficacies of the miR-140-5p mimic and inhibitor were verified by real-time PCR (Fig. 4.G). To determine the role of Fxr1 in PAH, the Fxr1 mRNA and protein expressions were detected in PA tissues and HPASMCs which demonstrated that Fxr1 was significantly upregulated (Fig. 5. C-F). Together, Fxr1 may be a target gene of miR-140-5p and may be involved in PAH progression.

\section{PVT1/ miR-140-5p/Fxr1 signaling axis regulated PAH progression}

Based on the observations, PVT1, miR-140-5p, and Fxr1 may form a signaling axis to regulate PAH progression. Thus, mRNA and protein levels of PVT1 in sh-PVT1-trasnfected HPASMCs were detected which showed that PVT1 was downregulated at both mRNA and protein levels (Fig. 6.A and B). Furthermore, sh-PVT1-induced downregulation of PVT1 was enhanced and reversed by the miR-140-5p mimic and inhibitor, respectively (Fig. 6.C and D). Functionally, sh-PVT1-induced inhibitory effect on HPASMCs proliferation and migration was enhanced and offset by miR-140-5p mimic and inhibitor, respectively (Fig. 7.A and B). The similar effects of the miR-140-5p mimic and inhibitor on cell proliferation was found in sh-PVT1-treated HPASMCs, as detected by the flow cytometer (Fig. 7.C). Western blots revealed that cell proliferation marker expressions were decreased in HPASMCs treated with the sh-PVT1 and miR-140-5p mimic whereas HPASMCs transfected with the sh-PVT1 and miR-140$5 p$ inhibitor did not alter (Fig. 7.D). Collectively, the PVT1/ miR-140-5p/Fxr1 signaling axis may be essential for the regulation of cell proliferation and migration in HPASMCs.

\section{Discussion}

Pulmonary arterial hypertension (PAH) is defined as a devastating disease of the small pulmonary arteries manifesting aberrant vascular proliferation and remodeling $[29,30]$. Pulmonary vascular remodeling, along with vasoconstriction and thrombosis in situ, are three primary factors leading to the elveated pulmonary vascular resistance [4]. As a complex pathological process, pulmonary vascular remodeling is characterized by the disrupted HPASMC proliferation and migration, as well as the inhibited apoptosis. Therefore, exploring the molecular and cellular mechanism underlying HPASMC proliferation and apoptosis in PAH would greatly contribute to the understanding of PAH pathology. In the present 
study, the results demonstrated that the expression of PVT1 was increased in PA tissues and HPASMCs of patients with PAH and its expression was positively correlated with pulmonary artery pressure. In addition, the knockdown and overexpression of PVT1 inhibited and promoted HPASMC proliferation and migration, respectively. Furthermore, bioinformatics analysis and functional studies showed that the effect of PVT1 on HPASMC cellular activities in PAH may be mediated by the miR-140-5p/Fxr1 signaling axis.

The IncRNA PVT1 is 1716 nucleotides in length and is encoded by an oncogene PVT1 [31]. It has been reported that PVT1 exerts essential roles in various types of tumors, including hepatocellular carcinoma [32], malignant pleural mesothelioma[33], bladder cancer [34], and gastric cancer [13], where PVT1 suppresses apoptosis while promoting cell proliferation and migration, eventually facilitating cancer progression [12]. Since PASMCs and cancer cells share some common cellular properties, such as hypermetabolism, aberrant mitochondrial function, and high proliferative activity [35, 36], PVT1 may also function as a stimulator for PASMC proliferation and migration. In this study, PVT1 was upregulated in both PA tissues and HPASMCs. Functional studies revealed that overexpression of PVT1 significantly elevated viability and proliferation of HPASMCs. The results are consistent with the hypothesis that PVT1 promotes pulmonary vascular remodeling by enhancing proliferation and inhibiting apoptosis, as its roles in several tumor types [12]. However, since the effect of PVT1 displays the MYC copy-number-dependent pattern, the mechanism underlying its effect may vary in each pathological process [37].

In addition to its role in cellular activities, this study found that the expression of PVT1 was positively correlated with pulmonary artery pressure, indicating PVT1 may be a promising diagnostic biomarker for patients with PAH. Similarly, PVT1 has been identified as an ideal prognostic biomarker in several cancer types [32, 38, 39], in which the upregulation of PVT1 is significantly correlated with poor overall survival, distant metastasis, and poor differentiation grade [19]. Therefore, more studies are needed to further determine the predictive value of PVT1 in PAH.

It has been well-documented that IncRNAs can function as ceRNA to regulate the expression of microRNAs (miRNAs), primarily through sponging and silencing miRNAs [40,41]. Therefore, to further determine the mechanism underlying the effect of PVT1 on PAH, bioinformatics analysis was performed to screen the putative miRNAs interacting with PVT1. Combining with the mechanic and functional studies, the results revealed that the expression of PVT1 was negatively correlated with the level of miR140-5p and their physical and functional interactions played important roles in the regulation of PAH progression, suggesting that PVT1 may act as a ceRNA of miR-140-5p in PAH. As a multifunctional factor, miR-140-5p exerts essential roles in various pathological processes, such as osteogenesis [42], epithelial mesenchymal transition [43], and autophagy [44]. A comprehensive bioinformatics analysis also demonstrated that miR-140-5p was associated with 23 target genes and seven signaling pathways in multiple biological processes, such as signal transduction and cell proliferation [45]. The expression of miR-140-5p is downregulated in PAH and the suppression of miR-140-5p facilitates proliferation and migration in HPASMCs [46], which agrees with the observation in the present study. As such, like PVT1, miR-140-5p may also serve as a potential biomarker for PAH.

Page 10/21 
In this study, the results also demonstrated that Fxr1 was upregulated in both PA tissues and HPASMCs and its effect on vascular remodeling was opposite to those of miR-140-5p. These results together indicated that miR-140-5p may act as a sponge of Fxr1 in PAH, which was confirmed by bioinformatics analysis and luciferase reporter assays. The Fxr1 is an RNA-binding protein and its sequence is highly conservative across many species $[47,48]$. Like the roles of RNA-binding proteins, Fxr1 is associated with gene translation, mRNA transport, and mRNA binding through G4 RNA structures or AU-rich elements [48, 49]. On the basis of modulation of cellular activities, Fxr1 participates in the regulation of p21 expression via p53-dependent patterns to regulate cell proliferation in the head and neck squamous cell carcinoma and non-small cell lung cancer cells [50]. Also, Fxr1 functions as an oncogene promoting proliferation, invasion, as well as migration in cancer cells [51]. Together, Fxr1 may be an important regulator of cell proliferation and migration.

In this study, we have demonstrated that the important role of PVT1 in the HPASMCs of PAH. However, some limitations in this study should not be ignored. For example, the animal experiment should be perfomed to validate the effect of PVT1 in vivo. Also, the pathogenesis of PAH is heterogeneous [52, 53] and there are several subtypes of PAH, such as idiopathic PAH (IPAH), heritable PAH (HPAH), and associated PAH (APAH) [54]. Of which, we only included HPASMCs of HPAH in the present study. Thus, more subtype of PAH should be covered in future study.

In conclusion, PVT1 was upregulated in PA tissues and HPASMCs and the expression of PVT1 was positively correlated with pulmonary artery pressure. Also, PVT1 could promote proliferation while inhibiting apoptosis in HPASMCs though the miR-140-5p/Fxr1 signaling axis. Understanding the molecular mechnisms of PVT1 in PAH may provide novel insight into developing therapy for PAH.

\section{Conclusions}

In conclusion, PVT1 was upregulated in PA tissues and HPASMCs and the expression of PVT1 was positively correlated with pulmonary artery pressure. Also, PVT1 could promote proliferation while inhibiting apoptosis in HPASMCs though the miR-140-5p/Fxr1 signaling axis. Understanding the molecular mechnisms of PVT1 in PAH may provide novel insight into developing therapy for PAH.

\section{Abbreviations}

Pulmonary arterial hypertension (PAH)

long non-coding RNAs (IncRNAs)

plasmacytoma variant translocation 1 (PVT1)

pulmonary artery smooth muscle cells (HPASMCs)

\section{Declarations}




\section{Ethics approval and consent to participate}

This experiment has got the approval of the Medical Ethics Committee of Cangzhou Central Hospital and this study is in line with the Declaration of Helsinki.

\section{Consent for publication}

Not applicable

\section{Availability of data and materials}

The datasets used and/or analysed during the current study are available from the

\section{Competing interests}

The authors declare that they have no competing interests

\section{Conflicts of Interest}

The authors declare no conflict of interest

\section{Funding}

This research received no external funding

\section{Author Contributions}

Botao Zhao performed the majority of experiments and analyzed the data; Yuchao Shen performed the molecular investigations; Qinghua Wu designed and coordinated the research; Li Yao and Yamin Hu wrote the paper.

\section{Acknowledgment}

None

References 
1. Humbert M, Sitbon O, Simonneau G. Treatment of pulmonary arterial hypertension. N Engl J Med. 2004;351(14):1425-36.

2. Archer SL, Weir EK, Wilkins MR. Basic science of pulmonary arterial hypertension for clinicians: new concepts and experimental therapies. Circulation. 2010;121(18):2045-66.

3. Bienertova-Vasku J, Novak J, Vasku A. MicroRNAs in pulmonary arterial hypertension: pathogenesis, diagnosis and treatment. Journal of the American Society of Hypertension: JASH. 2015 Mar;9(3):221-34.

4. Voelkel N. Pathophysiology of primary pulmonary hypertension: from physiology to molecular mechanisms. Primary pulmonary hypertension. 1997.

5. Humbert M, Morrell NW, Archer SL, et al. Cellular and molecular pathobiology of pulmonary arterial hypertension. J Am Coll Cardiol. 2004;43(12 Supplement):13-24.

6. Pietra GG, Capron F, Stewart S, et al. Pathologic assessment of vasculopathies in pulmonary hypertension. J Am Coll Cardiol. 2004;43(12 Supplement):25-32.

7. Perros F, Dorfmüller P, Souza R, et al. Fractalkine-induced smooth muscle cell proliferation in pulmonary hypertension. Eur Respir J. 2007;29(5):937-43.

8. McMurtry MS, Bonnet S, Wu X, et al. Dichloroacetate prevents and reverses pulmonary hypertension by inducing pulmonary artery smooth muscle cell apoptosis. Circulation research. 2004;95(8):83040.

9. Schmitt AM, Chang HY. Long noncoding RNAs in cancer pathways. Cancer cell. 2016;29(4):452-63.

10. Shi X, Sun M, Liu H, et al. Long non-coding RNAs: a new frontier in the study of human diseases. Cancer letters. 2013;339(2):159-66.

11. Fatica A, Bozzoni I. Long non-coding RNAs: new players in cell differentiation and development. Nat Rev Genet. 2014;15(1):7.

12. Cui M, You L, Ren X, et al. Long non-coding RNA PVT1 and cancer. Biochem Biophys Res Commun. 2016;471(1):10-4.

13. Ghafouri-Fard S, Omrani MD, Taheri M. Long noncoding RNA PVT1: A highly dysregulated gene in malignancy. J Cell Physiol. 2020;235(2):818-35.

14. Chen J, Yu Y, Li H, et al. Long non-coding RNA PVT1 promotes tumor progression by regulating the miR-143/HK2 axis in gallbladder cancer. Mol Cancer. 2019;18(1):33.

15. He F, Song Z, Chen $\mathrm{H}$, et al. Long noncoding RNA PVT1-214 promotes proliferation and invasion of colorectal cancer by stabilizing Lin28 and interacting with miR-128. Oncogene. 2019;38(2):164-79.

16. Wang D, Hu Y. Long non-coding RNA PVT1 competitively binds microRNA-424-5p to regulate CARM1 in radiosensitivity of non-small-cell lung cancer. Molecular Therapy-Nucleic Acids. 2019;16:130-40.

17. Wang Z, Zhang Q, Sun Y, et al. Long Non-Coding RNA PVT1 Regulates BAMBI To Promote Tumor Progression In Non-Small Cell Lung Cancer By Sponging miR-17-5p. Onco Targets Ther. 2020;13:131.

18. Zheng J, Hu L, Cheng J, et al. IncRNA PVT1 promotes the angiogenesis of vascular endothelial cell by targeting miR-26b to activate CTGF/ANGPT2. Int J Mol Med. 2018;42(1):489-96. 
19. Association WM. World Medical Association Declaration of Helsinki. Ethical principles for medical research involving human subjects. Bull World Health Organ. 2001;79(4):373.

20. Galiè N, Humbert M, Vachiery J-L, et al. 2015 ESC/ERS guidelines for the diagnosis and treatment of pulmonary hypertension: the Joint Task Force for the Diagnosis and Treatment of Pulmonary Hypertension of the European Society of Cardiology (ESC) and the European Respiratory Society (ERS): endorsed by: Association for European Paediatric and Congenital Cardiology (AEPC), International Society for Heart and Lung Transplantation (ISHLT). Eur Heart J. 2016;37(1):67-119.

21. Mao W, Xia W, Chen J, et al. Interobserver variability in grading acute rejection after lung transplantation. Chest. 2014;145(2):416-7.

22. Meloche J, Le Guen M, Potus F, et al. miR-223 reverses experimental pulmonary arterial hypertension. Am J Physiol Cell Physiol. 2015 Sep 15;309(6):C363-72.

23. Livak KJ, Schmittgen TD. Analysis of relative gene expression data using real-time quantitative PCR and the 2(-Delta Delta C(T)) Method. Methods. 2001 Dec;25(4):402-8.

24. Pulito C, Mori F, Sacconi A, et al. Metformin-induced ablation of microRNA 21-5p releases Sestrin-1 and CAB39L antitumoral activities. Cell discovery. 2017;3:17022.

25. Rasband W. ImageJ software. Bethesda: US National Institutes of Health; 2011.

26. Liu X-h, Sun M, Nie F-q, et al. Lnc RNA HOTAIR functions as a competing endogenous RNA to regulate HER2 expression by sponging miR-331-3p in gastric cancer. Mol Cancer. 2014;13(1):92.

27. Noguchi T, Dobashi Y, Minehara H, et al. Involvement of cyclins in cell proliferation and their clinical implications in soft tissue smooth muscle tumors. Am J Pathol. 2000;156(6):2135-47.

28. Hui W, Yuntao L, Lun L, et al. MicroRNA-195 inhibits the proliferation of human glioma cells by directly targeting cyclin D1 and cyclin E1. PLoS One. 2013;8(1):e54932.

29. Rubin LJ. Primary pulmonary hypertension. N Engl J Med. 1997;336(2):111-7.

30. Runo JR, Loyd JE. Primary pulmonary hypertension. The lancet. 2003;361(9368):1533-44.

31. Lu D, Luo P, Wang Q, et al. IncRNA PVT1 in cancer: A review and meta-analysis. Clin Chim Acta. 2017:474:1-7.

32. Ding C, Yang Z, Lv Z, et al. Long non-coding RNA PVT1 is associated with tumor progression and predicts recurrence in hepatocellular carcinoma patients. Oncology letters. 2015;9(2):955-63.

33. Riquelme E, Suraokar MB, Rodriguez J, et al. Frequent coamplification and cooperation between CMYC and PVT1 oncogenes promote malignant pleural mesothelioma. Journal of Thoracic Oncology. 2014;9(7):998-1007.

34. Zhuang C, Li J, Liu Y, et al. Tetracycline-inducible shRNA targeting long non-coding RNA PVT1 inhibits cell growth and induces apoptosis in bladder cancer cells. Oncotarget. 2015;6(38):41194.

35. Leopold JA, Maron BA. Molecular Mechanisms of Pulmonary Vascular Remodeling in Pulmonary Arterial Hypertension. Int J Mol Sci. 2016;17(5):761.

36. Bonnet S, Rochefort G, Sutendra G, et al. The nuclear factor of activated T cells in pulmonary arterial hypertension can be therapeutically targeted. Proceedings of the National Academy of Sciences. 
2007;104(27):11418-11423.

37. Tseng Y-Y, Moriarity BS, Gong W, et al. PVT1 dependence in cancer with MYC copy-number increase. Nature. 2014;512(7512):82.

38. Cui D, Yu C-H, Liu M, et al. Long non-coding RNA PVT1 as a novel biomarker for diagnosis and prognosis of non-small cell lung cancer. Tumor Biology. 2016;37(3):4127-34.

39. Gao J, Cao R, Mu H. Long non-coding RNA UCA1 may be a novel diagnostic and predictive biomarker in plasma for early gastric cancer. Int J Clin Exp Pathol. 2015;8(10):12936.

40. Gibb EA, Brown CJ, Lam WL. The functional role of long non-coding RNA in human carcinomas. Mol Cancer. 2011;10(1):38.

41. Ebert MS, Sharp PA. MicroRNA sponges: progress and possibilities. Rna. 2010;16(11):2043-50.

42. Hwang S, Park S-K, Lee HY, et al. miR-140-5p suppresses BMP2-mediated osteogenesis in undifferentiated human mesenchymal stem cells. FEBS Lett. 2014;588(17):2957-63.

43. Lv J, Fan H-x, Zhao X-p, et al. Long non-coding RNA Unigene56159 promotes epithelialmesenchymal transition by acting as a ceRNA of miR-140-5p in hepatocellular carcinoma cells. Cancer letters. 2016;382(2):166-75.

44. Zhai $\mathrm{H}$, Fesler $\mathrm{A}, \mathrm{Ba} \mathrm{Y}$, et al. Inhibition of colorectal cancer stem cell survival and invasive potential by hsa-miR-140-5p mediated suppression of Smad2 and autophagy. Oncotarget. 2015;6(23):19735.

45. Li F, Shi W, Wan Y, et al. Prediction of target genes for miR-140-5p in pulmonary arterial hypertension using bioinformatics methods. FEBS Open Bio. 2017;7(12):1880-90.

46. Rothman AM, Arnold ND, Pickworth JA, et al. MicroRNA-140-5p and SMURF1 regulate pulmonary arterial hypertension. J Clin Investig. 2016;126(7):2495-508.

47. Kirkpatrick LL, Mcllwain KA, Nelson DL. Comparative genomic sequence analysis of the FXR gene family: FMR1, FXR1, and FXR2. Genomics. 2001;78(3):169-77.

48. Vasudevan S, Steitz JA. AU-rich-element-mediated upregulation of translation by FXR1 and Argonaute 2. Cell. 2007;128(6):1105-18.

49. Schaeffer $C$, Bardoni $B$, Mandel JL, et al. The fragile $X$ mental retardation protein binds specifically to its mRNA via a purine quartet motif. EMBO J. 2001;20(17):4803-13.

50. Majumder $\mathrm{M}$, House R, Palanisamy N, et al. RNA-binding protein FXR1 regulates p21 and TERC RNA to bypass p53-mediated cellular senescence in OSCC. PLoS Genet. 2016;12(9):e1006306.

51. Jin X, Zhai B, Fang T, et al. FXR1 is elevated in colorectal cancer and acts as an oncogene. Tumor Biology. 2016;37(2):2683-90.

52. Meloche J, Le Guen M, Potus F, et al. miR-223 reverses experimental pulmonary arterial hypertension. American Journal of Physiology-Cell Physiology. 2015;309(6):C363-72.

53. Yuan JX-J, Rubin LJ. Pathogenesis of pulmonary arterial hypertension: the need for multiple hits. Am Heart Assoc; 2005.

54. Machado RD, Eickelberg O, Elliott CG, et al. Genetics and genomics of pulmonary arterial hypertension. J Am Coll Cardiol. 2009;54(1 Supplement):32-42. 

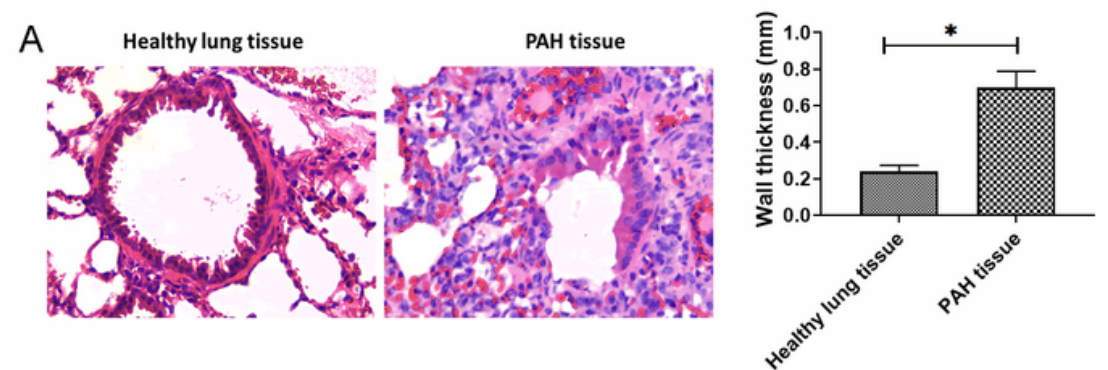

C

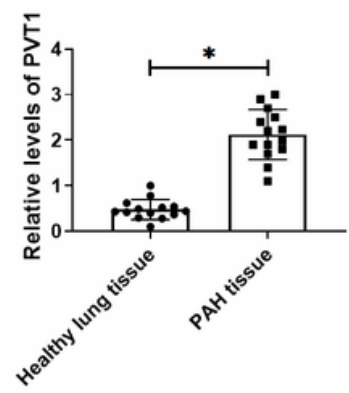

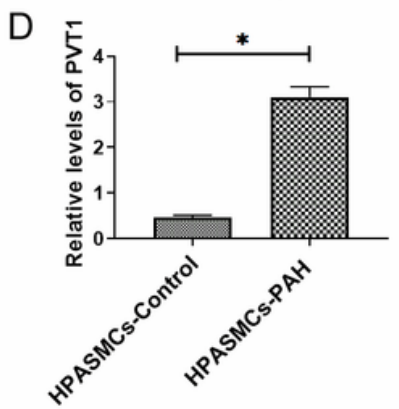

$\mathrm{E}$

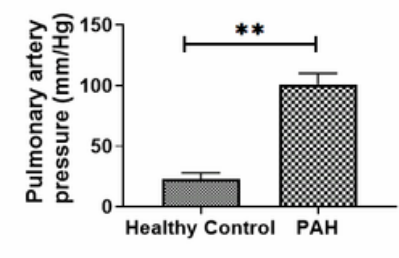

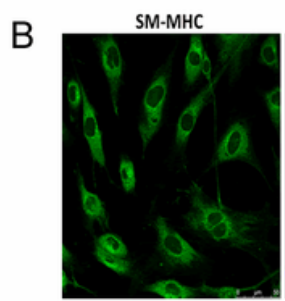
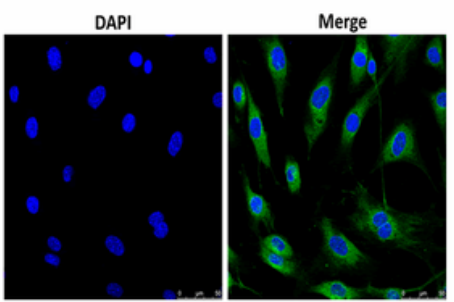

$\mathrm{F}$

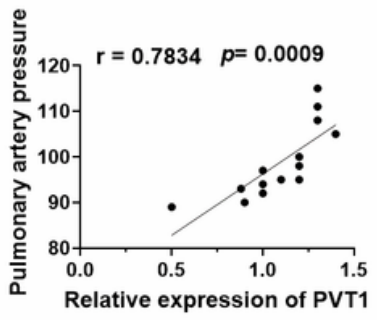

\section{Figure 1}

Upregulation of PVT1 in PA tissues and HPASMCs. (A) Hematoxylin \& eosin staining of human pulmonary artery samples (scale bar=50 $\mu \mathrm{m}$ ) and the vascular wall thickness. (B) Immunofluorescence assay of smooth muscle myosin heavy chain in HPASMCs. (C). The expression level of PVT1 in PA tissues. (D) The expression level of PVT1 in HPASMCs. (E) Pulmonary artery pressure in patients with $\mathrm{PAH}$ and control participants. (F) Pearson's correlation analysis for expression of PVT1 and pulmonary artery pressure. ${ }^{*} \mathrm{P}<0.05,{ }^{*} \mathrm{P}<0.01,{ }^{*} * \mathrm{P}<0.001$.
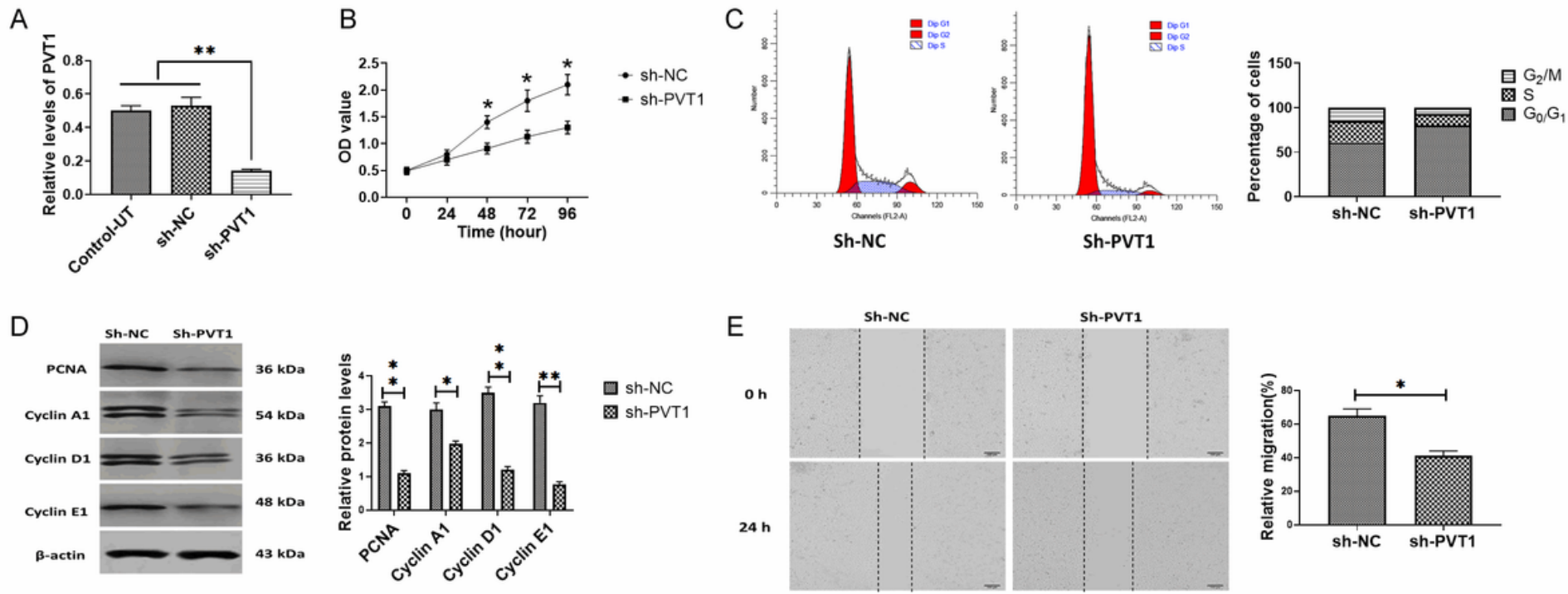

Figure 2 
Downregulation of PVT1 inhibited HPASMCs proliferation and migration. (A). Transfection efficacy of shPVT1. (B) Cell vitality in HPASMCs transfected with sh-PVT1, as detected by CCK-8 assays. (C). Cell proliferation in HPASMCs transfected with sh-PVT1, as detected by flow cytometer assays. (D) Protein expressions of PCNA, Cyclin A1, Cyclin D1, and Cyclin E1 in HPASMCs transfected with sh-PVT1, as detected by western blots. $(E)$. Cell migration ability in HPASMCs transfected with sh-PVT1, as detected by wound-healing migration assays. ${ }^{*} P<0.05, * * P<0.01, * * * P<0.001$.

A

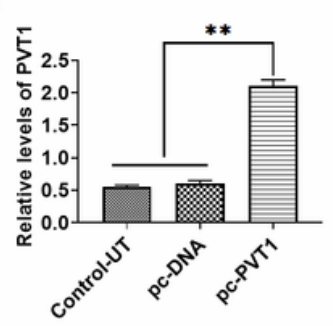

D

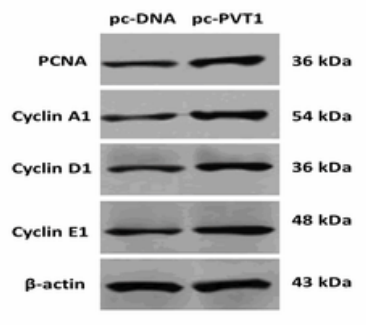

B
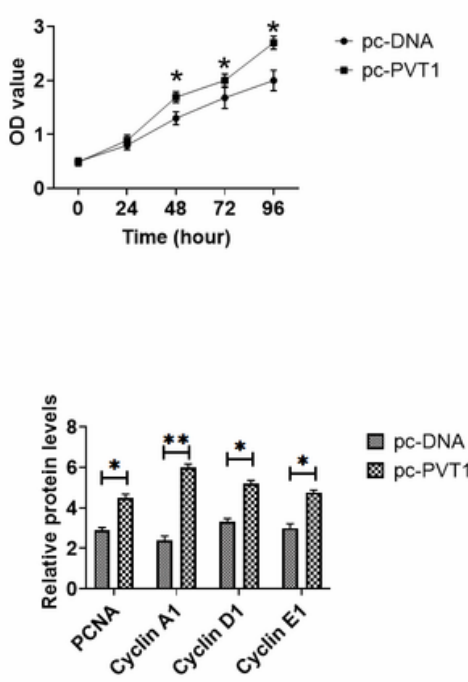

C

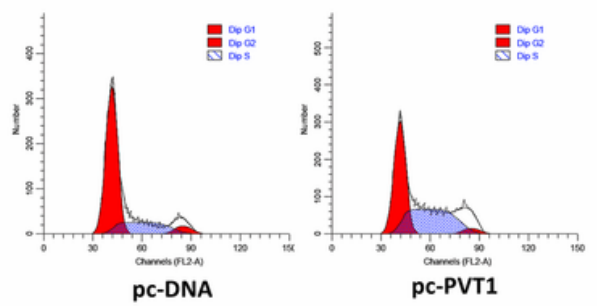

$\mathrm{E}$

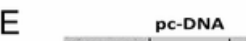

on

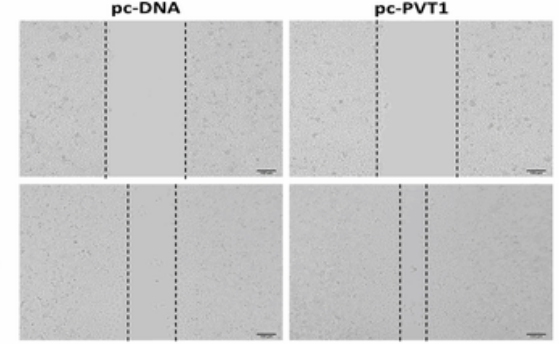

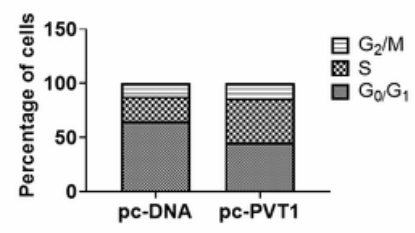

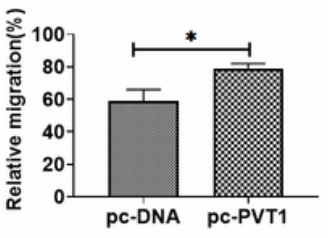

Figure 3

Upregulation of PVT1 promoted HPASMCs proliferation and migration. (A). Transfection efficacy of pcPVT1. (B) Cell vitality in HPASMCs transfected with pc-PVT1, as detected by CCK-8 assays. (C). Cell proliferation in HPASMCs transfected with pc-PVT1, as detected by flow cytometer assays. (D) Protein expressions of PCNA, Cyclin A1, Cyclin D1, and Cyclin E1 in HPASMCs transfected with pc-PVT1, as detected by western blots. (E). Cell migration ability in HPASMCs transfected with pc-PVT1, as detected by wound-healing migration assays. $* P<0.05, * * P<0.01, * * * P<0.001$. 
A

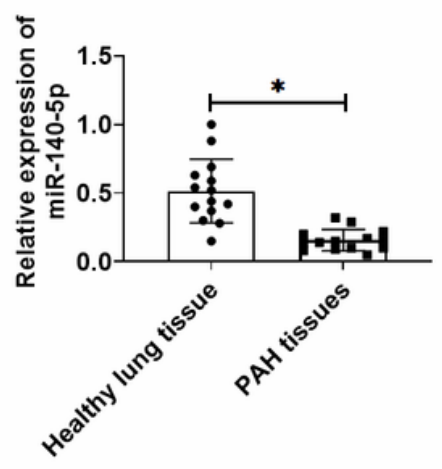

E

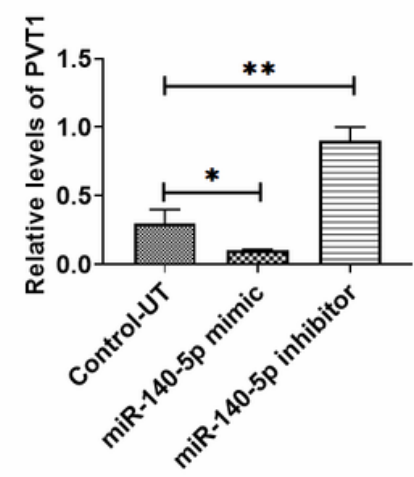

$\mathrm{H}$

PVI1: 5 ' gaccgcuuucacACGCAGCCUg 3' (Mutant)

II

PVI1: 5 ' gaccgcuuucacAAAACCACUg 5' (Wildtype) $\||||||| \mid$

miR-140-5p : 3' gaugguaucccaUUJUGGUGAc 5'
B

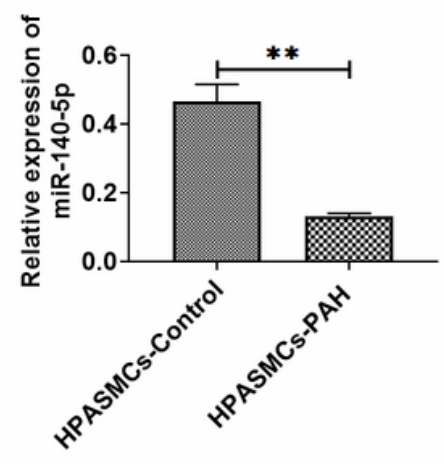

F

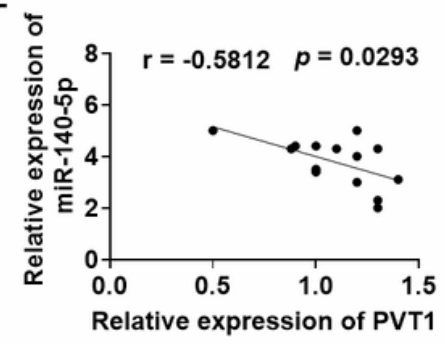

C เึิ
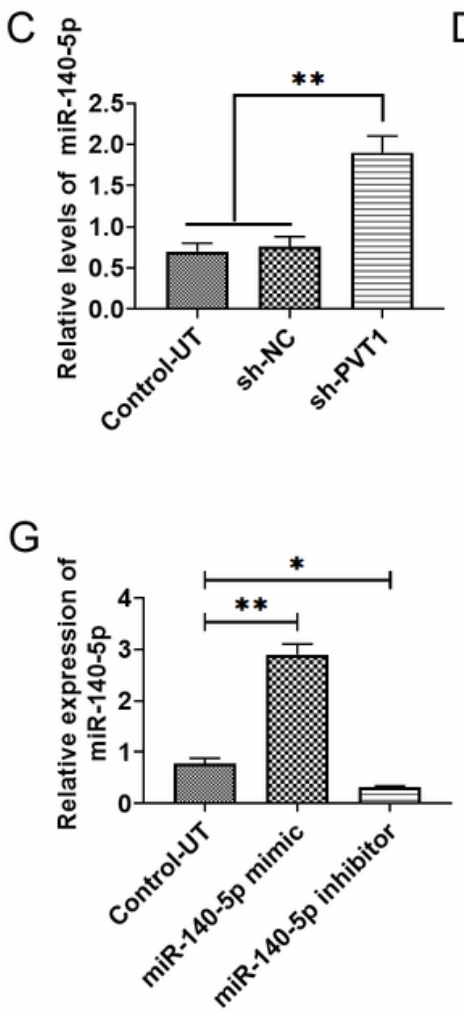

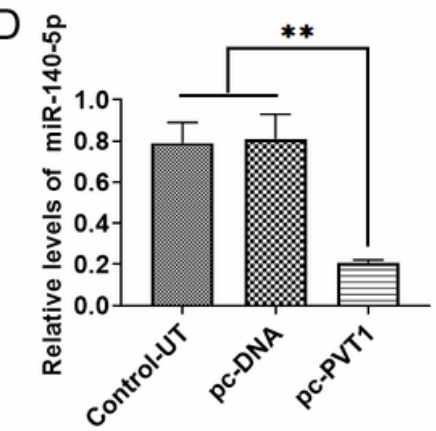

$\mathrm{J}$
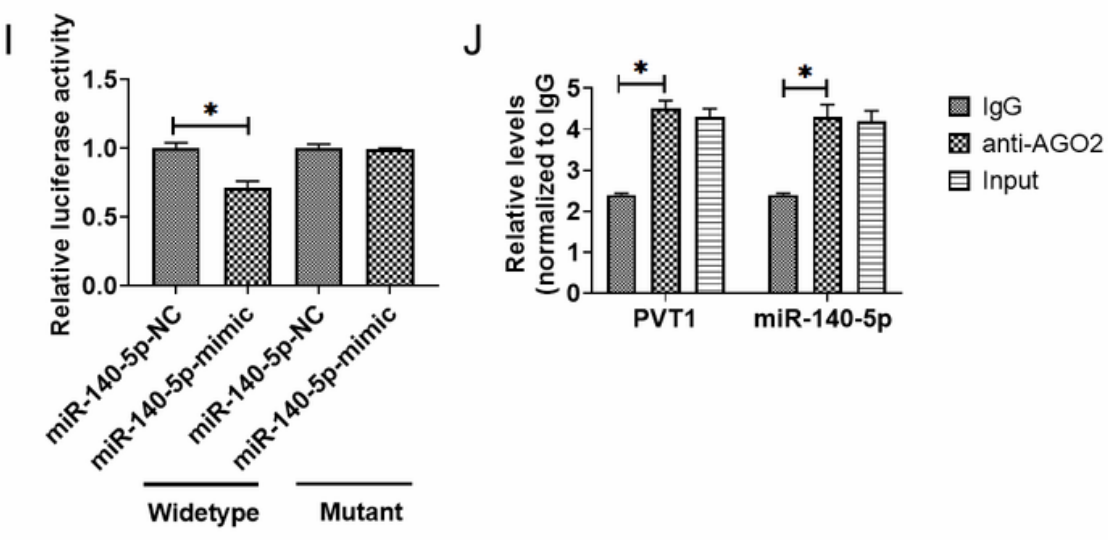

\section{Figure 4}

PVT1 acted as sponge for miR-140-5p in HPASMCs. (A). The expression level of miR-140-5p in PA tissues. (B) The expression level of miR-140-5p in HPASMCs. (C) The expression level of miR-140-5p in HPASMCs transfected with sh-PVT1. (D) The expression level of miR-140-5p in HPASMCs transfected with pc-PVT1. (E). The expression level of PVT1 in HPASMCs transfected with the miR-140-5p mimic and inhibitor. (F). Pearson's correlation analysis for expressions of PVT1 and miR-140-5p. (G). The transfection efficacies of the miR-140-5p mimic and inhibitor. $(\mathrm{H})$ Putative miR-340-5p binding site in the 3'UTR of PVT1. (I) Interaction between PVT1 and miR-140-5p was determined by luciferase reporter assays. (J) Interaction between PVT1 and miR-140-5p was determined by RNA immunoprecipitation assays. ${ }^{*} P<0.05, * * P<0.01, * \star * P<0.001$. 

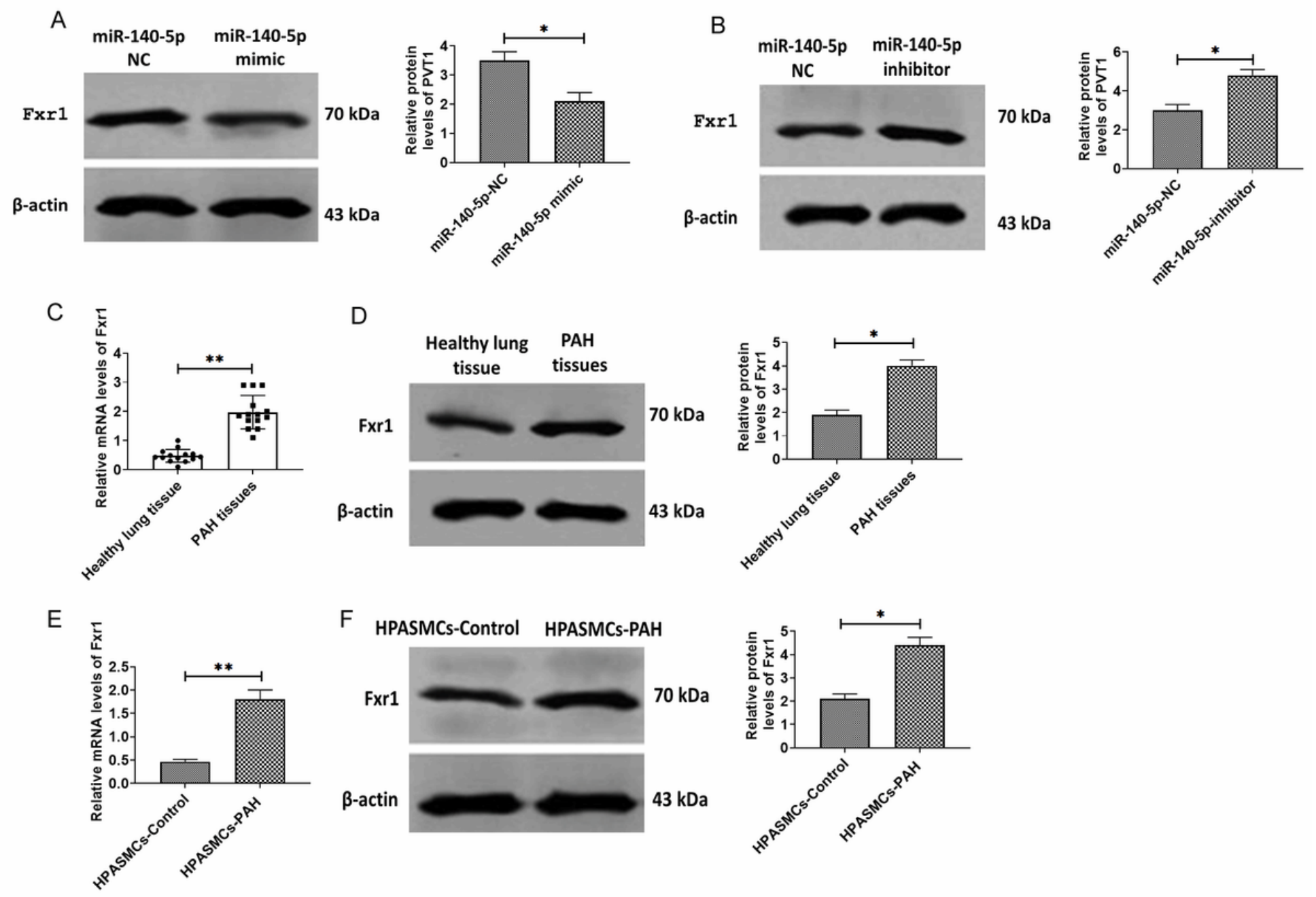

G

Fxr1: 5' cauuuuCAagCAaguCCCACUu 3' (Mutant) II:

Fxr1: 5' cauuuuUAAUAUGCAACCACUu 3' (widetype) II |: || || ||

miR-140-5p: 3' gaugguAUCCCAUUUUGGUGAC 5'

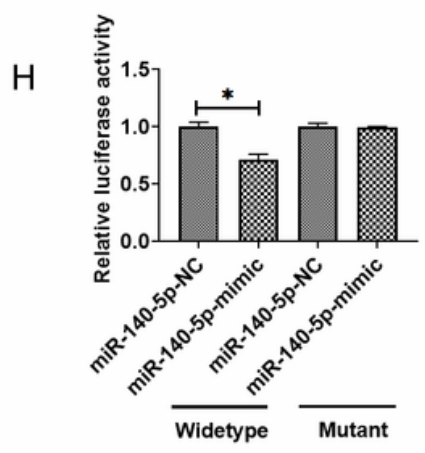

\section{Figure 5}

Fxr1 was a target gene of miR-140-5p in HPASMCs. (A). Protein expression of Fxr1 in HPASMCs transfected with the miR-140-5p mimic. (B) Protein expression of Fxr1 in HPASMCs transfected with the miR-140-5p inhibitor. (C-D). The mRNA and protein expression of Fxr1 in PA tissues. (E-F) The mRNA and protein expression of Fxr1 in HPASMCs. (G). Putative miR-340-5p binding site in the 3'UTR of Fxr1. (H) Interaction between miR-140-5p and Fxr1 was determined by luciferase reporter assays. ${ }^{*} P<0.05$, ${ }^{\star *} \mathrm{P}<$ $0.01, * \star * P<0.001$. 

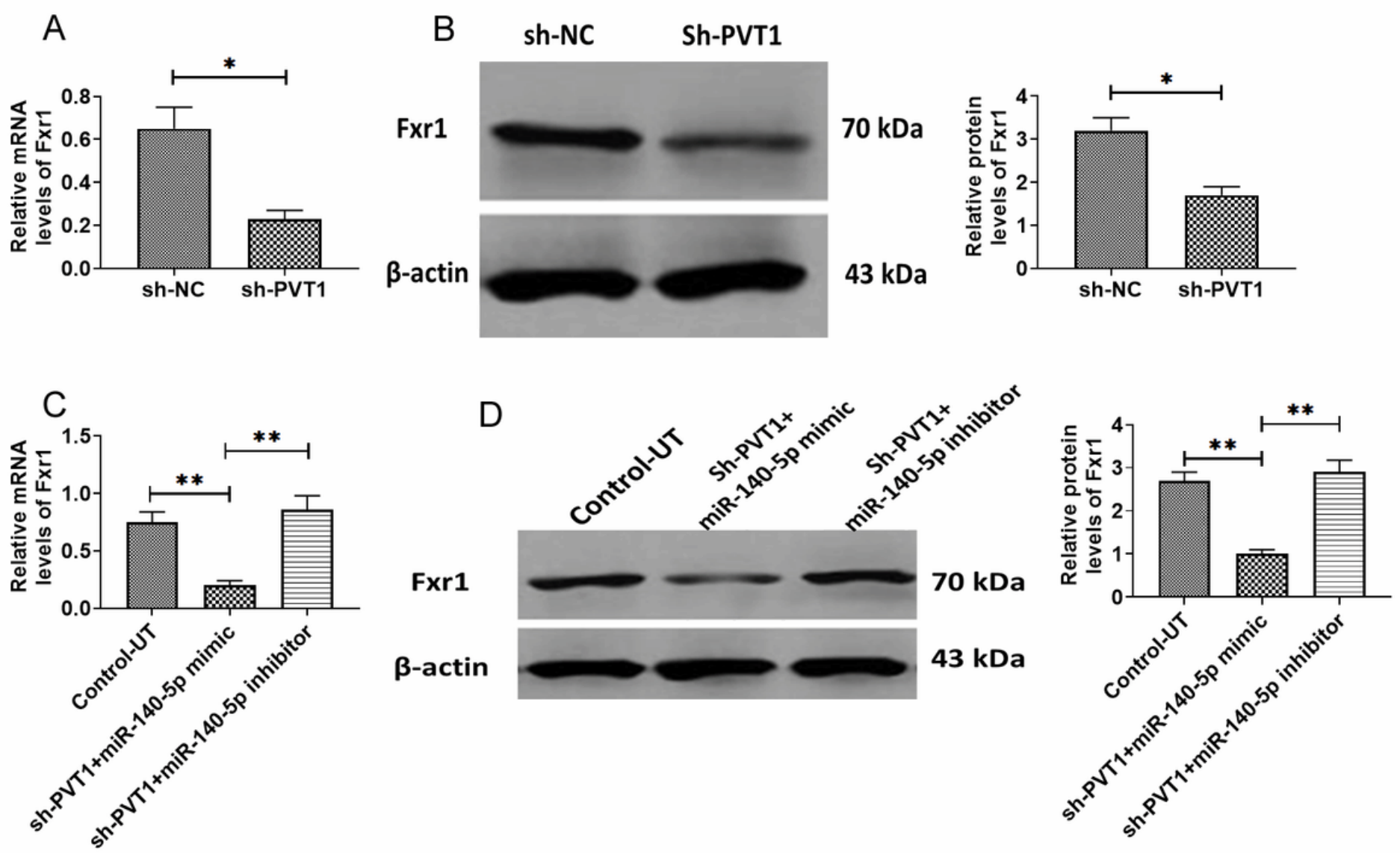

\section{Figure 6}

Fxr1 was associated with the PVT1/miR-140-5p signaling axis. (A-B) The mRNA and protein expression of Fxr1 in HPASMCs transfected with sh-PVT1. (C-D). The mRNA and protein expression of Fxr1 in shPVT1-treated HPASMCs transfected with the miR-140-5p mimic and inhibitor. ${ }^{\star} P<0.05,{ }^{\star *} P<0.01, * \star \star P$ $<0.001$. 

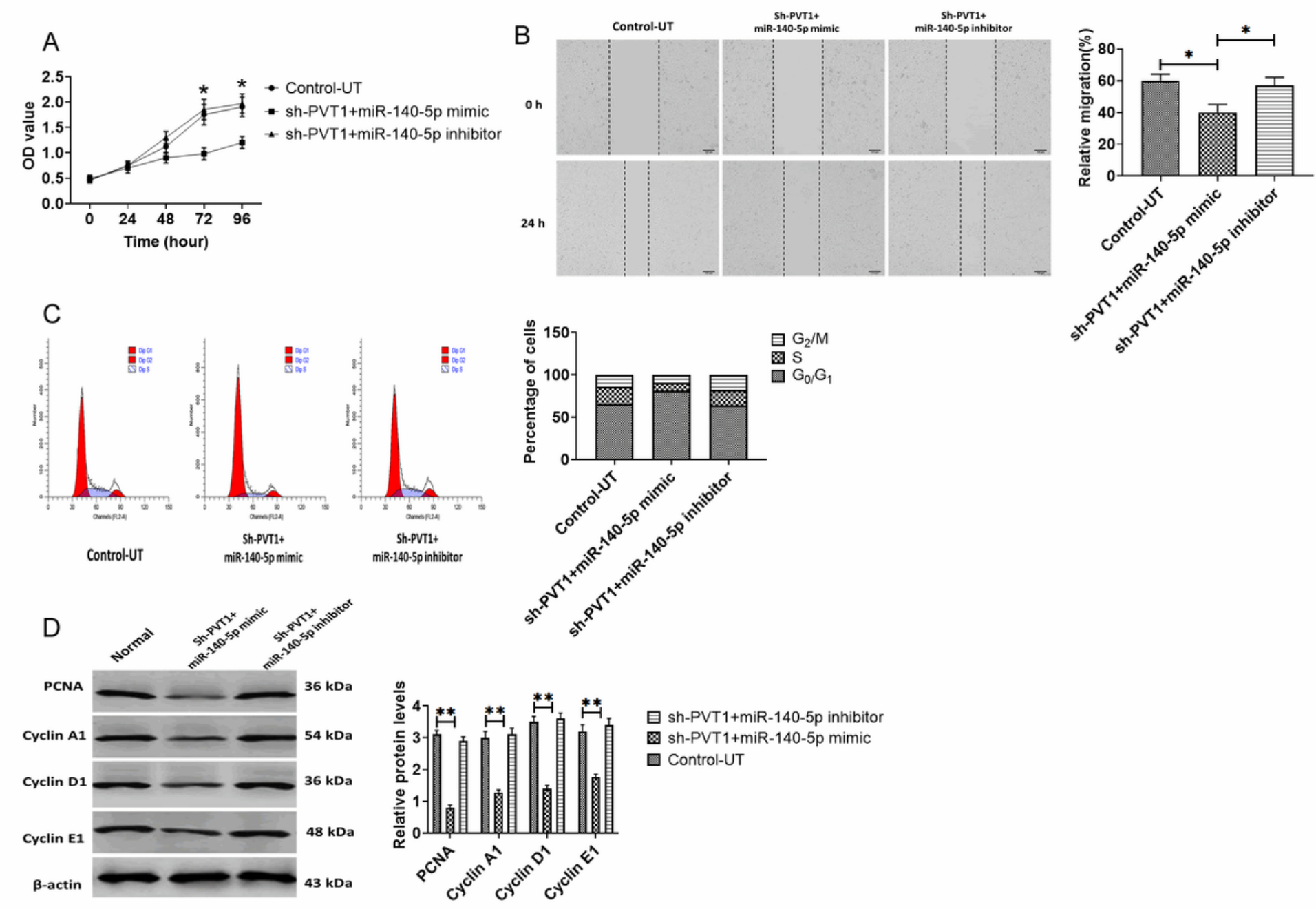

Figure 7

The PVT1/ miR-140-5p/Fxr1 signaling axis regulated PAH progression. (A). Cell vitality in sh-PVT1-treated HPASMCs transfected with the miR-140-5p mimic and inhibitor, as detected by CCK-8 assays. (B). Cell migration ability in sh-PVT1-treated HPASMCs transfected with the miR-140-5p mimic and inhibitor, as detected by wound-healing migration assays. (C) Cell proliferation in sh-PVT1-treated HPASMCs transfected with the miR-140-5p mimic and inhibitor, as detected by flow cytometer assays. (D) Protein expressions of PCNA, Cyclin A1, Cyclin D1, and Cyclin E1 in sh-PVT1-treated HPASMCs transfected with the miR-140-5p mimic and inhibitor, as detected by western blots. ${ }^{*} P<0.05,{ }^{*} \mathrm{P}<0.01,{ }^{\star * *} \mathrm{P}<0.001$. 